\title{
Cooperación en seguridad entre los Estados miembros del CCG: el impacto que supone la emergencia del Estado Islámico
}

\author{
Cooperação em segurança entre os Estados membros do CCG: o impacto diante da \\ emergência do Estado Islâmico
}

Ornela Fabani ${ }^{1}$

\begin{abstract}
RESUMEN
El objetivo del trabajo reside en analizar cómo ha evolucionado la cooperación en materia de seguridad entre los países miembros del CCG y qué impacto ha tenido sobre la misma la emergencia del Estado Islámico. Mientras tanto, nuestro supuesto de partida radica en que, pese a las limitaciones que ha demostrado la cooperación en esta materia entre los miembros del bloque, a partir del surgimiento del Estado Islámico se avizora un relanzamiento de la misma que responde en gran medida al temor a esta amenaza.
\end{abstract}

Palabras clave: Consejo de Cooperación de Estados Árabes del Golfo; cooperación en seguridad; Estado Islámico

\begin{abstract}
The objective of this paper is to analyze how security cooperation between GCC members has evolved and what impact the emergence of the Islamic State has had on it. Meanwhile, our starting assumption is that, despite the limitations that security cooperation among GCC members has demonstrated, since the emergence of the Islamic State we can find a relaunch of it that largely responds to the fear of this threat.
\end{abstract}

Key words: Gulf Cooperation Council; security cooperation; Islamic State

1 Becaria posdoctoral del Consejo Nacional de Investigaciones Científicas y Técnicas (CONICET). Doctora en Relaciones Internacionales por la Universidad Nacional de Rosario. Magíster en Integración y Cooperación Internacional, Centro de Estudios Interdisciplinarios, Universidad Nacional de Rosario. Rosario, Santa Fe, Argentina. 


\section{Introducción}

El Consejo de Cooperación de Estados Árabes del Golfo (CCG) es un organismo subregional integrado por Bahrein, Kuwait, Omán, Qatar, Arabia Saudita y Emiratos Árabes Unidos, que se conformó el 25 de mayo de 1981. Al respecto, resulta interesante subrayar que a diferencia de otros bloques que se constituyen netamente con vistas a lograr una mejor inserción de sus socios en el mercado internacional, el CCG reviste la particularidad de que sus miembros impulsaron su conformación con el fin último de reunir fuerzas para afrontar conjuntamente una serie de amenazas que los acechaban (Fabani, 2012a).

De hecho, podemos afirmar que aquello que movilizó a las monarquías del Golfo, impulsándolas a dar vida a un organismo subregional, fue su búsqueda de asegurar su supervivencia frente a una serie de amenazas externas con las cuales estos países debieron lidiar (Fabani, 2012b). Esto explica cómo un grupo de Estados cuyos regímenes políticos se caracterizan por ser jerárquicos, centralizados, fuertemente personalistas, y que, precisamente por poseer estas particularidades, son aún más reacios que otros Estados a delegar cuotas de poder; decidieron cooperar en un campo tan sensible a la soberanía estatal como lo es aquel de la seguridad.

Entre finales de los setenta y principios de los ochenta las amenazas a la seguridad de estos países eran múltiples y giraban en torno a: la presencia de fuerzas soviéticas en Afganistán, la revolución islámica que pretendía exportar Irán y la guerra entre Irán e Irak; despertando enorme preocupación entre los Estados del Golfo que temían por su supervivencia. Básicamente, en un mundo anárquico en el marco del cual no existe una autoridad central y donde el conflicto es latente, la supervivencia emerge como el fin último de todo Estado. En consonancia, siguiendo a Waltz (1988: 186), el objetivo último que persiguieron estos Estados al tomar la decisión de crear este bloque no fue maximizar su poder -como sostendría Morgenthau (1963)- sino tomar medidas en pos de su seguridad y, en última instancia, velar por su supervivencia.

Ahora bien, en lo que respecta al CCG, más allá de los embates de la cooperación en seguridad entre los miembros del bloque y del declive que esta sufre una vez culminada la primera década de vida de este organismo, lo cierto es que al conjugarse la emergencia del 
Estado Islámico con otras amenazas a la seguridad y la estabilidad regional estos países deciden relanzar la misma con las vistas puestas, una vez más, en su propia salvaguardia.

Tras esta breve introducción, el objetivo general de nuestro trabajo reside en analizar cómo ha evolucionado la cooperación en materia de seguridad entre los países miembros del CCG y qué impacto ha tenido la emergencia del Estado Islámico sobre la misma. Mientras tanto, nuestro supuesto de partida radica en que, pese a las limitaciones que ha demostrado la cooperación en materia de seguridad entre los miembros del CCG, a partir del surgimiento del Estado Islámico se avizora un relanzamiento de la misma que responde en gran medida al temor a esta amenaza. Dicho esto, en un primer apartado damos cuenta del devenir de la cooperación en materia de seguridad entre los seis miembros del bloque a través del tiempo para, luego y teniendo en consideración la experiencia previa, atender a las diversas respuestas que han ensayado estas naciones ante la aparición del Estado Islámico, evaluando particularmente el correlato que ha tenido su surgimiento sobre la cooperación en materia de seguridad entre los socios del citado esquema.

\section{Avances y retrocesos de la cooperación en seguridad entre los miembros del CCG}

Teniendo en consideración el complejo escenario regional en el marco del cual se constituyó el CCG, los miembros del bloque comenzaron a transitar el camino de la cooperación en materia de seguridad a escasos meses de haberse dado vida a este organismo. Tal es así que durante la segunda mitad de 1981 prosperaron las reuniones de Jefes de Estado Mayor, Ministros de Defensa y Ministros del Interior, esencialmente como respuesta a los avatares de la guerra entre Irán e Irak (Ramazani, Kechichian, 1988: 60 y ss).

En lo que respecta a los resultados de estos encuentros, a raíz de los mismos las partes decidieron desarrollar una serie de ejercicios militares conjuntos en pequeña escala que involucraron tanto sus fuerzas aéreas como terrestres; los primeros en la historia entre estos seis Estados vecinos (Ramazani, Kechichian, 1988: 60 y ss). Asimismo, tras dichas maniobras se manifestó necesario unificar los métodos de entrenamiento y la currícula de las academias militares. En esa época también se trabajó en la posibilidad de integrar sistemas de radares aéreos y terrestres y en la creación de un sistema aéreo de alarma y de control combinado. 
Por otra parte, Arabia Saudita conjuntamente con Kuwait brindaron asistencia a Bahrein para que éste pudiera modernizar su flota de aviones de combate y a su vez contar con una moderna base aérea. Es más, a mediados de los ochenta, el Consejo Supremo, órgano máximo del bloque, decidió otorgar un préstamo de 2 billones de dólares a Omán para que este estuviese en condiciones de modernizar su sistema de defensa y de incrementar la seguridad de la nueva red de radares en el estrecho de Ormuz (Fabani, 2012a: 83 y ss).

De cualquier forma, el gran hito en lo que respecta a la cooperación en materia de seguridad entre las partes se concretó en 1984, cuando se arribó a la conformación de la fuerza militar conjunta del bloque denominado "Peninsula Shield Force" (PSF) (Fabani, 2012b). Esta fuerza, que en su composición inicial tenía un carácter netamente terrestre, para 1985 reunía alrededor de unos siete mil soldados y tenía su sede en la ciudad militar de King Khaled, Arabia Saudita (Kuffel, 2000: 5).

La reflexión insondable respecto a la conformación de esta fuerza gira en torno a la originalidad de la misma, pues su creación se convierte en un hecho sin precedentes para la historia de la integración regional². Más aún, llama la atención que los países que impulsaron la constitución del PSF son Estados monárquicos, que se sustentan en regímenes personalistas, donde existe una fuerte centralización del poder en torno a la figura del Rey. De allí que sólo podemos comprender cabalmente su decisión de avanzar en este sentido al considerar los peligros que, entonces, las monarquías del Golfo debieron afrontar.

Ahora bien, más allá de estos prometedores avances iniciales y aunque los miembros del CCG dieron un paso de la envergadura de la conformación del PSF, llegando incluso a sostener en diferentes encuentros que un ataque contra cualquiera de los miembros del bloque era un ataque contra todos; lo cierto es que este principio fue relegado al ámbito meramente discursivo cuando uno de los Estados parte del bloque tras ser atacado necesitó de su aplicación. Esto se evidencia al constatar que la fuerza militar conjunta de los Estados del Golfo no intervino como hubiese sido de esperar al momento de la invasión a Kuwait. De

\footnotetext{
2 En efecto, al hacer memoria podemos citar como otro ejemplo en esta dirección al proyecto para conformar una Comunidad Europea de Defensa que, como bien es sabido, fue una idea impulsada en la década del cincuenta por los seis países parte de la, entonces, Comunidad Económica del Carbón y del Acero -Francia, Alemania Occidental, Bélgica, Países Bajos, Italia y Luxemburgo- que, sin embargo, finalmente no prosperó dado que la Asamblea Nacional Francesa rechazó la ratificación del acuerdo que le daba origen.
} 
hecho, en aquel entonces, los Estados miembros del CCG se limitaron a condenar el accionar de Irak sin lograr coordinar una acción conjunta de la fuerza del bloque.

Quien interviene entonces liberando al pequeño emirato, invadido y luego anexado por Irak, es una fuerza internacional compuesta por más de 30 naciones, liderada por Estados Unidos y bajo mandato de Naciones Unidas. La guerra del Golfo se transformó entonces en un punto de inflexión para el organismo subregional en lo que atañe a la cooperación en materia de seguridad; pues a partir de la misma estos Estados dejaron de lado el optimismo inicial en torno a sus posibilidades de acción conjunta y parecieron reconocer sus propias limitaciones para hacerse cargo de su propia seguridad.

De hecho, una vez culminada la guerra, los miembros del CCG recién volvieron a sentirse seguros tras la firma de una serie de acuerdos de cooperación defensiva con Estados Unidos. Tal es así que la etapa que entonces se inició se caracterizó por una notable profundización del vínculo con Washington, dando paso a la presencia directa de efectivos norteamericanos, el preposicionamiento de equipo militar, el incremento de las ventas de armamento, el desarrollo de ejercicios conjuntos, la profundización de la asesoría, y el establecimiento de bases aéreas y navales en la región (Mesa Delmonte, 2002: 77). Lo expuesto pone en evidencia que, más allá de los esfuerzos realizados por los Estados del Golfo en lo ateniente a la cooperación en seguridad durante la primera década de vida del bloque, éstos pasaron a depender, una vez más, de una potencia extra regional antes que de sus pares del CCG como garante de su seguridad.

Ahora, cabe mencionar que la confianza en el rol que a partir de entonces asumió Estados Unidos terminó siendo contraproducente para el devenir de la cooperación en materia de seguridad entre los miembros del organismo. Decimos esto pues estos Estados, al depositar su confianza en el apoyo norteamericano, culminaron descuidando la cooperación entre sí, la cual se fue diluyendo a la par que se profundizaban los vínculos bilaterales con Washington. Es decir, emerge una relación dialéctica donde el rol que Estados Unidos aún hoy juega en la región es producto de las dificultades que las seis monarquías han encontrado para cooperar entre sí pero, asimismo, es un factor que ha contribuido al debilitamiento de la cooperación en seguridad entre las mimas.

En lo que respecta al devenir de la cooperación en materia de seguridad entre los miembros del CCG a posteriori de la invasión a Kuwait, ante todo hemos de advertir que, 
nuevamente, los esfuerzos se concentraron en torno al "Peninsula Shield Force". Lo que resulta comprensible si consideramos que la conformación y evolución de esta fuerza puede pensarse como el mayor de los logros del bloque en lo que respecta a la cooperación en esta área. Tal es así que culminada la guerra del Golfo surgieron múltiples propuestas respecto a cuál debería de ser su destino (Fabani, 2012b).

Sin ir más lejos, en la Cumbre de Jefes de Estado del CCG que tuvo lugar en Qatar en 1991 Omán propuso reforzar la fuerza al punto de que la misma pasase a contar con cien mil efectivos. De igual forma, se sugirió que la comandancia de la misma rotase entre los Estados parte. No obstante, no logró concitarse un acuerdo al respecto. Luego, en 1993 se retomó el proyecto de ampliar la fuerza. Esta vez la idea era que la misma pasase a contar con veinticinco mil efectivos. Finalmente, en 1999, la discusión giró en torno a que el PSF dispusiera al menos de cinco mil soldados (Kuffel, 2000: 8).

Otra cuestión que merece atención a la hora de considerar los esfuerzos realizados por las monarquías del Golfo en lo que atañe a la cooperación en seguridad post guerra del golfo, es la firma por parte de estos Estados de un acuerdo de defensa en el año 2000. Respecto al contenido de este instrumento jurídico, el mismo otorgó status legal al compromiso previo de las partes de defenderse colectivamente sobre la base del principio de que un ataque contra cualquier miembro del CCG es un ataque contra todos (Koch, 2010: 8).

Ahora, siguiendo con los avatares del PSF, en 2006 se decidió que los efectivos apostados en la base Hafr Al Batin retornasen a sus respectivos países. La idea era que la fuerza sólo conservase una estructura administrativa, por esto se estipuló que se conformaría un comando central bajo la égida de la Secretaria General en Riad que se encargaría de hacer un llamamiento a las unidades militares de cada Estado cuando fuese necesario recurrir a las mismas frente a una emergencia (Kahwahi, 2008).

Dos años después se discutió la posibilidad de convertir a esta fuerza en un aparato de reacción rápida que pasase a contar con veintidós mil efectivos (Kahwahi, 2008) y que, asimismo, dispusiese de capacidades aéreas y navales de las cuales hasta entonces carecía; una propuesta que finalmente se aprobó en la Cumbre de Jefes de Estado de 2009.

Por último, en mayo de 2011, al conmemorarse treinta años de la conformación del CCG, una vez más se reflotó la idea de ampliar el PSF (Kermali, 2011). Al respecto, llama nuestra atención que la ampliación y el devenir de esta fuerza siga siendo objeto de discusión 
entre los miembros del bloque, cuando la realidad es que el accionar de la misma ha sido no sólo secundario sino incluso podríamos decir que inexistente en aquellos momentos en los que alguno de estos Estados tuvo que hacer frente a una amenaza externa. Léase: frente a la invasión de Irak a Kuwait en 1990; tras la acción de Irán que despojó a Emiratos Árabes Unidos de tres islas en 1992 o, inclusive, ante el ataque de Irak a Kuwait en el marco de la invasión de Estados Unidos en 2003. Como consecuencia, concordamos con Legrenzi (2006: 12) en que el rol de esta fuerza a través del tiempo ha sido básicamente simbólico.

\section{Desafíos y respuestas a la emergencia del Estado Islámico}

En lo que hace a las características actuales del escenario de Medio Oriente, hoy en día la situación en la región se muestra sumamente compleja. Esto en virtud de una serie de conflictos o focos de tensión que han hecho eclosión en la zona y que nos permiten concluir que este espacio atraviesa por una etapa de fuerte inestabilidad. Entre ellos vale mencionar: el conflicto con los Houthis ${ }^{3}$ en Yemen; la creciente influencia de Irán en diversos países de la región y el debate que su programa de desarrollo nuclear suscita; y la fragmentación estatal en Siria, Irak y Libia, que se han convertido en terreno fértil para el accionar de organizaciones terroristas y a su vez para la proliferación de milicias armadas. Finalmente, en relación directa con este último punto, no podemos dejar de mencionar la avanzada del Estado Islámico que se presenta como una amenaza a la integridad territorial y la soberanía de los países sobre los cuales se ha afincado. De hecho, este actor presenta un importantísimo desafío a la seguridad regional, amén de haberse convertido, sino en la mayor amenaza, en una de las principales amenazas a la seguridad de los miembros del CCG.

Al respecto, si bien podríamos conjeturar que entre los países miembros del bloque aquellos que en mayor medida se ven amenazados por el Estado Islámico son Arabia Saudita y Kuwait, particularmente en virtud de su proximidad geográfica a la frontera con Irak; lo cierto es que esta entidad representa una amenaza para el conjunto de las monarquías del

\footnotetext{
3 Movimiento originario de la provincia de Saada, en Yemen, que en septiembre 2014 logró hacerse del control de la capital de aquel país para luego anunciar la destitución del presidente Abd Rabbuh Mansur. 
Golfo, porque el desafío que este supone tiene un fuerte componente ideológico. Decimos esto ya que el Estado Islámico ha proclamado un califato islámico y ha manifestado que los musulmanes de todo el mundo le deben lealtad al mismo. Aún más, su líder Abubakr al Baghdadi, quien se abroga el título de Califa, ha subrayado que él y su organización constituyen la única autoridad legítima de la comunidad islámica (Nalapat, 2014).

De hecho, pese a que el accionar y las ideas que abraza el Estado Islámico sean desdeñadas por gran parte de los musulmanes, su prédica podría suponer un grave reto a la paz y la seguridad en las monarquías del Golfo. En este sentido, es necesario recordar que el grueso de la población en los países miembros del CCG es joven (Nalapat, 2014), así como también lo son gran parte de los adherentes al Estado Islámico, y que la influencia del wahabismo ${ }^{4}$ en estas naciones conlleva que estos países puedan convertirse en terreno fértil para que allí prendan los argumentos teológicos de este grupo.

En este marco, entre los Estados parte del bloque existe la preocupación de que elementos radicales puedan vincularse con los grupos de oposición existentes al interior de sus respectivos países o establecer grupos subversivos en la Península Arábiga, incrementando el peligro de un ataque a figuras de alto nivel y a las instalaciones petroleras. Otra amenaza potencial que cobra peso con el Estado Islámico reside en la profundización de las tensiones sectarias en aquellos países del CCG que tienen una población shiíta sustancial: Arabia Saudita (20\%), Kuwait (30\%) y Bahrein (70\%) (Guzansky, 2014). Como consecuencia, los miembros del CCG han ensayado distintas respuestas a la amenaza que supone el surgimiento de este actor. Entre ellas podemos dar cuenta de una serie de acciones individuales que emprendieron estos países.

Sin ir más lejos, las fuerzas de seguridad saudíes han detenido a supuestos activistas del Estado Islámico que presuntamente reclutaban adherentes a la organización y recolectaban donaciones en dinero y armas, amén de encargarse de la planificación de ataques en todo el Reino. Asimismo, se ha dado a conocer un decreto real imponiendo severos castigos a todo aquel que se una, financie o se identifique con la organización (Guzansky, 2014). Al respecto, esta medida resulta paradojal si atendemos a que existe información que

\footnotetext{
${ }^{4}$ Corriente ortodoxa del Islam que se desprende de la vertiente sunita.
} 
da cuenta que las monarquías del Golfo, ya sea de forma directa o indirecta, financiaron a grupos radicales en Siria con el objetivo de derrocar al régimen de Bahasr Al Assad ${ }^{5}$, los cuales se convirtieron en el germen del Estado Islámico.

Por otra parte, el gobierno saudí ha intensificado su control sobre los predicadores, así como también sobre los contenidos que circulan en Internet, y ha iniciado una campaña en pos de la deslegitimación ideológica del Estado Islámico a través de programas de televisión y artículos de prensa (Prahan, 2015: 4). Otra acción que demuestra la creciente amenaza planteada por el Estado Islámico, reside en la decisión de Arabia Saudita de apostar 30.000 efectivos militares en su frontera con Irak. Además, el Reino se encuentra avanzando en la construcción de una barrera de $900 \mathrm{~km}$ para fortificar su frontera norte (Prahan, 2015: 6). Es más, ante el temor de Riad a que jóvenes saudíes se vean tentados a ingresar a las filas del Estado Islámico, particularmente atendiendo al alto desempleo y a la frustración que golpea a muchos de ellos en el país, los Al Saud han introducido programas y aumentado el financiamiento para este colectivo en el ámbito de la vivienda, el empleo y la educación superior (Guzansky, 2014).

Además, tanto el Reino saudita como Emiratos Árabes Unidos han introducido legislación antiterrorista que ha recibido duras críticas por parte de organizaciones defensoras de los derechos humanos, atendiendo a que la definición de terrorismo a la cual estas recurren abarca diversas formas de activismo político e intelectual. Como correlato se teme que estas leyes puedan resultar aplicadas contra miembros de la oposición que critiquen a estos gobiernos o se manifiesten a favor de una reforma política.

En cuanto a Bahrein, allí se ha arrestado, condenado y, en algunos casos, despojado de la ciudadanía a bahreiníes acusados de apoyar al Estado Islámico (Katzman, 2015c: 30). Si bien, también en este caso, existen entidades que denuncian que esta amenaza está siendo utilizada como una excusa contra aquellos que buscan hacer oír sus voces al interior del Reino. Amén de ello, Manama también está reforzando las medidas de seguridad alrededor de las mezquitas en virtud de las amenazas efectuadas por el Estado Islámico. Allí grupos de

\footnotetext{
5 Ver: Carrión, Francisco, Los bolsillos que financian el terror del ISIS, El Mundo, 24/06/2014. Disponible en: http://www.elmundo.es/internacional/2014/06/24/53a99799e2704e13298b4584.html, Islamic State: where does jihadists group gets it support?, $B B C, 01 / 09 / 2014$. Disponible en: http://www.bbc.com/news/world-middle-east-29004253 
voluntarios entrenados por las fuerzas de defensa civil se han desplegado en diferentes partes del país para apoyar la labor de vigilancia los días viernes, cuando un gran número de musulmanes acuden a los espacios de oración (Atassi, 2015).

De cualquier forma, más allá de considerar estas acciones individuales, en este caso nos interesa particularmente atender al impacto de la emergencia del Estado Islámico sobre la cooperación en seguridad entre los miembros del CCG; pues a partir de la aparición de esta entidad se avizora un relanzamiento de la misma que responde en gran medida al temor a esta nueva amenaza.

En este sentido, hay quienes definen al Estado Islámico como un super pegamento que ha impulsado el accionar conjunto de los miembros del CCG (Young, 2014). De hecho, todo indica que el desafío que ha supuesto su emergencia así como su expansión, se ha convertido en un factor central que ha favorecido que los seis miembros de la organización se vuelquen, una vez más, a la cooperación en materia de seguridad superando incluso la crisis diplomática ${ }^{6}$ que golpeó al bloque a lo largo de 2014 y que implicó que Emiratos Árabes, Arabia Saudita y Bahrein retirasen sus embajadores de Doha, en un hecho que no tiene precedentes en la historia del bloque. Tal es así que en la XXXV Cumbre del CCG que tomó lugar en diciembre de 2014 los representantes de las monarquías del Golfo volvieron a insistir en la cooperación en materia de seguridad y acordaron el establecimiento de un comando militar conjunto que tendrá su sede en la ciudad de Riad.

Ahora bien, es importante no confundir al mismo con el PSF. De hecho, a diferencia de la estructura original del PSF, el comando unificado cuya conformación se acordó en la citada cumbre no tendrá una fuerza permanente. Por lo contrario, el mismo comprenderá unidades especiales apostadas en establecimientos militares existentes en cada país miembro del CCG que podrán ser convocadas cuando resulte necesario (Duke Anthony, 2014: 1).

Asimismo, vale mencionar que en el citado encuentro también se concertó la creación de una fuerza policial regional, además de una fuerza naval conjunta. En torno a la fuerza policial del bloque, que recibe el nombre de GCC-POL (atendiendo a la sigla en inglés

\footnotetext{
${ }^{6}$ Crisis desatada como producto de las desavenencias suscitadas entre los miembros del bloque a partir de las políticas divergentes por estos asumidas ante los conflictos latentes en la región de Medio Oriente, y muy especialmente, a raíz del vínculo y la percepción que cada uno de ellos guarda frente a una agrupación de la características de Hermanos Musulmanes.
} 
con las que se hace referencia al CCG) es importante atender a las declaraciones de altas autoridades del bloque que han mencionado que se apuesta a que este cuerpo pueda mejorar la cooperación contra el terrorismo. En esta misma dirección, se espera que esta fuerza contribuya a coordinar esfuerzos en materia de inteligencia y a compartir conocimientos e información entre las autoridades pertinentes para eventualmente racionalizar los esfuerzos en materia de seguridad (Vela, 2014).

Finalmente, se prevé que la fuerza naval del bloque se asiente en Bahrein. Aún más, con vistas en la puesta en vigor de la misma en el mes de marzo, unidades de los seis países participaron de un ejercicio naval conjunto que se extendió por diez días (The National, 2015). Respecto al propósito del mismo, este consistió en elevar el nivel de preparación, la eficacia en combate y la cooperación entre las fuerzas de los seis países. Esto con el objetivo de lograr unificar las operaciones navales de los Estados del CCG y de intercambiar experiencias para hacer de esta marina una fuerza de disuasión en la región (Ghazanfar, 2015).

Frente al efectivo relanzamiento de la cooperación en seguridad entre los miembros del CCG cabe preguntarnos por qué los seis socios del bloque insisten en crear un comando unificado y una fuerza naval conjunta teniendo en consideración el escaso alcance que previamente ha tenido el PSF. Especialmente considerando que el accionar de esta última ha sido inexistente en aquellos momentos en los que alguno de estos Estados tuvo que hacer frente a una amenaza externa a su seguridad. Al respecto, todo indica que los seis socios del CCG tienen interés en continuar mostrándose como un frente unido no sólo en el ámbito externo, sino también en el plano interno; apostando a que el comando militar conjunto, la fuerza naval y el GCC POL actúen como elementos disuasorios al menos para repeler posibles ataques terroristas al interior de estos Estados. Es más, podemos conjeturar que seguir dando difusión al debate en torno al devenir de las fuerzas del CCG y los ejercicios militares conjuntos, posiblemente sirva incluso a las autoridades de estos Estados para camuflar frente a la opinión pública la presencia en sus territorios de efectivos militares provenientes de diversos países occidentales. 


\section{La participación de los miembros del CCG en la coalición contra el Estado Islámico y la evolución del vínculo con Washington}

Ahora bien, lo cierto es que la decisión de los miembros del bloque de dar impulso, una vez más, a la cooperación en materia de seguridad, de ninguna forma supuso la intención de las monarquías del Golfo de tomar distancia de Estados Unidos, de cobrar independencia del paraguas de seguridad que este país les brinda. Por el contrario, en paralelo tanto a las acciones individuales emprendidas como a aquellas consensuadas intra-bloque, los países del CCG han continuado cultivando sus vínculos con Washington. En esta línea, las seis naciones del Golfo se han sumado a la coalición internacional liderada por dicho país contra el Estado Islámico.

La adhesión a la misma se concretó tras la participación de estas monarquías de una reunión en Jeddah, en septiembre de 2014, en la cual también estuvieron presentes el Secretario de Estado de Estados Unidos y representantes de otros cuatro países árabes Egipto, Irak, Jordania, Líbano-. En efecto, en el marco de dicho encuentro se firmó un comunicado conjunto en el que las partes manifestaron estar "unidas contra la amenaza que representa el terrorismo, incluyendo el llamado Estado Islámico" (Karasik, 2014). Amén de ello, por medio del citado documento los referidos actores acordaron detener el flujo de combatientes extranjeros a través de los países vecinos, luchar contra aquellos que financian al Estado Islámico y otros extremistas violentos, contribuir en los esfuerzos en pos de brindar ayuda humanitaria a las poblaciones más afectadas por el Estado Islámico, colaborar con la reconstrucción y la rehabilitación de las comunidades dañadas por el citado actor y, finalmente, unirse a la campaña militar coordinada contra el mismo (Karasik, 2014).

Respecto a este último punto, Arabia Saudita, Bahrein, Qatar, y Emiratos Árabes han participados de los bombardeos sobre el territorio de Siria controlado por el Estado Islámico. Entre estos países, Emiratos Árabes Unidos ha llevado a cabo más ataques en Siria que cualquier otro miembro de la coalición con excepción de Estados Unidos, siendo el único Estado árabe al que Washington le ha permitido comandar ataques aéreos (Katzman, 2015b: 20).

En otro orden, estas naciones han puesto a disposición sus instalaciones y han realizado importantes aportes económicos en términos de ayuda humanitaria. En cuanto a 
las bases militares, se estima que las fuerzas norteamericanas están haciendo uso de $\mathrm{Al}$ Dhafra en Emiratos Árabes, Ali Al Salem en Kuwait y Al Udeid en Qatar (Chandrasekaran, 2014; Katzman, 2015a; Katzman, 2015b). En efecto, esta última es el centro neurálgico regional de la campaña contra el Estado Islámico. La misma funge como un espacio donde se coordina el accionar de la coalición, se intercambia información y se planifican las misiones (Chandrasekaran, 2014).

Por otra parte, Emiratos Árabes Unidos ha recibido en su territorio a las fuerzas de otros países que participan en el esfuerzo contra el Estado Islámico, incluyendo aviones franceses estacionados en la base aérea de Al Dhafra y componentes de las fuerzas australianas. Además, junto con Alemania, están liderando la labor de reconstrucción y estabilización de zonas de Irak liberadas previamente controladas por el Estado Islámico, lo que ha supuesto la creación de un fondo común para afrontar algunos de esos esfuerzos (Katzman, 2015b: 20).

Mientras tanto, si bien Kuwait no ha participado de los ataques aéreos contra el citado actor, ha autorizado el uso de sus bases e instalaciones militares a miembros de la coalición liderada por Estados Unidos permitiendo, incluso, a Canadá e Italia el estacionamiento de aviones de combate para su participación en la misión "Operación Resolución inherente" (Katzman, 2015a: 13). En otro orden, Kuwait ha encabezado los esfuerzos de los Estados del Golfo a la hora de ayudar a las víctimas civiles de los conflictos en Siria e Irak. En esta línea, ha organizado al menos dos conferencias de donantes en las que ha comprometido más de U\$ 600 millones en ayuda humanitaria (Katzman, 2015a: 19).

Si a lo hasta aquí expuesto agregamos que Estados Unidos ha decidido renovar el acuerdo de defensa que mantiene con Qatar, así como también ampliar las instalaciones de la base naval que posee en Bahrein y realizar importantes ventas de armas hacia estos países (Katzman, 2015c: 20 y ss), esto sin mencionar los compromisos asumidos por Obama en relación a seguridad de los mismos en la Cumbre de Camp David7 ; todo indica que, pese a los

\footnotetext{
${ }^{7}$ El presidente de los Estados Unidos se reunió con representantes de las monarquías del Golfo en la residencia presidencial de Camp David en mayo de 2015. Como resultado de este encuentro se dio a conocer un comunicado conjunto conforme con el cual "Estados Unidos está dispuesto a trabajar en forma conjunta con los Estados del CCG para disuadir y enfrentar a una amenaza externa a la integridad territorial de cualquier Estado del CCG". Para mayor información respecto a esta reunión ver: US assures GCC against external threat at summit. Middle East Monitor, 15/05/2015. Disponible en:
} 
resquemores que ha suscitado el acuerdo nuclear con Irán, la alianza entre estos actores continua firme.

\section{A modo de cierre}

Pese a las limitaciones que ha demostrado la cooperación en materia de seguridad entre los Estados miembros del CCG, a partir de la aparición del Estado Islámico se avizora un relanzamiento de la misma que responde en gran medida al temor a esta amenaza. De hecho, la insistencia en la cooperación en materia de seguridad por parte de los miembros del bloque sin lugar a dudas nos habla del enorme desafío que representa para estos seis países aquel que se erige, sino como el mayor, al menos como uno de los principales retos a la seguridad regional.

No obstante ello, la historia de este esquema en lo que atañe a la cooperación en materia de seguridad nos recuerda que avanzar en esta dirección no resultará fácil. De hecho, las expectativas que hoy generan los anuncios con respecto a la conformación del GCC POL, un comando militar unificado y una fuerza naval conjunta, decaen al atender a los avatares del PSF, que claramente no tuvo un accionar alentador.

En este marco, en base a la experiencia previa, todo indica que las nuevas entidades en cuya conformación hoy se trabaja bien podrían seguir el mismo derrotero que el PSF, esencialmente a raíz de la existencia de una serie de obstáculos que a través del tiempo han minado la cooperación en materia de seguridad entre los miembros del CCG y que hasta el momento el bloque no ha sabido sortear. Entre ellos: las dificultades que estos países han encontrado para consensuar cómo afrontar las principales amenazas a su seguridad; la inexistencia de una percepción compartida, que se vuelque en una postura conjunta frente a diversos conflictos regionales; y la desconfianza aún vigente entre las partes.

https://www.middleeastmonitor.com/news/americas/18644-us-assures-gcc-against-external-threats-atsummit 


\section{BIBLIOGRAFÍA}

ATASSI, Basma, Bahrain tightens security amid ISIL threats. Al Jazeera, 2015. Disponible en: http://www.aljazeera.com/news/2015/07/bahrain-tightens-security-isil-threats150703062006728 .html

CARRION, Francisco, Los bolsillos que financian el terror del ISIS. El Mundo, 2014. Disponible en: http://www.elmundo.es/internacional/2014/06/24/53a99799e2704e1 3298 b4 584.html

CHANDRASEKARAN, Rajiv, In the UAE, the US has a quiet potent ally called "Little Sparta". The National, 2014. Disponible en: https://www.washingtonpost.com/world/nationalsecurity/in-the-uae-the-united-states-has-a-quiet-potent-ally-nicknamed-littlesparta/2014/11/08/3fc6a50c-643a-11e4-836c-83bc4f26eb67_story.html

DUKE ANTHONY, John, Gulf Cooperation Council Establishes Unprecedented Joint Military Command. National Council on US-Arab Relations, 2014.

FABANI, Ornela (a), El Consejo de Cooperación de Estados Árabes del Golfo: un análisis de su evolución a 30 años de su conformación. Editorial Académica Española, Colección CERIR, Alemania, 2012.

FABANI, Ornela (b), Cooperación en seguridad entre las monarquías del Golfo: un análisis a tres décadas de la conformación del CCG, Cuadernos de Política Exterior Argentina, Centro de Estudios en Relaciones Internacionales de Rosario (CERIR), 2012.

GHAZANFAR, Ali Khan, GCC boosts drive to unify naval operations. Arab News, 2015. Disponible en: http://www.arabnews.com/featured/news/715336

GCC nations conduct joint naval exercise. The National, 2015. Disponible en: http://www.thenational.ae/uae/government/gcc-nations-conduct-joint-naval-exercise Islamic State: where does jihadists group gets it support?, $B B C, 2014$. Disponible en: http://www.bbc.com/news/world-middle-east-29004253

KAHWAJI, Riad, GCC creates quick reaction force. Defense News, 2008. Disponible en: http://www.defensenews.com/story.php?i=3354407, 2008.

KARASIK, Theodor, Analyzing the emergence of the GCC+4 against ISIS. Al Arabiya News, 2014. Disponible en: http://english.alarabiya.net/en/views/news/middleeast/2014/09/14/Analyzing-the-emergence-of-the-GCC-4-against-ISIS.html 
KATZMAN, Kenneth (a), Kuwait: Governance, Security and US policy. Congressional Research Service, 2015.

KATZMAN, Kenneth (b), The United Arab Emirates: Issues for US policy. Congressional Research Service, 2015.

KATZMAN, Kenneth (c), Bahrein: Reform, Security and US policy. Congressional Research Service, 2015.

KERMALI, Shenaz, The GCC is expanding its army, but for what? Aljazeera, 2011. Disponible en: http://www.aljazeera.com/indepth/features/2011/06/2011626112649845386.html KOCH, Kristian, The GCC as a regional security organization. KAS International Reports, Sankt Augustin, Nov. 10, Konrad Adenauer Stiftung e.V. Disponible en: http://www.kas.de/wf/doc/kas_21076-544-2-30.pdf?101110141517.

KUFFEL, Glenn, The Gulf Cooperation Council's Peninsula Shield Force. Naval War College, Newport, 2000. Disponible en: http://www.dtic.mil/cgi-bin/GetTRDoc?AD=ADA378521\& Location $=\mathrm{U} 2 \&$ doc $=$ GetTRDoc.pdf.

LEGRENZI, Matteo, The Peninsula Shield Force: End of a Symbol?. International Relations and Security Network, Issue $\mathrm{N}^{\circ} \quad 3,2006 . \quad$ Disponible en: http://www.isn.ethz.ch/isn/DigitalLibrary/Publications/Detail/?ots591=EB06339B-2726928E-0216-1B3F15392DD8\&lng=en\&id=56250.

MESA DELMONTE, Luis, EEUU en el golfo. Bases militares y sujeción política. Nación Árabe, $\mathrm{N}^{\circ}$ 47, Año XV, 2012.

MORGENTHAU, Hans, La Lucha por el Poder y por la Paz, Sudamericana, Buenos Aires, 1963. NALAPAT, M. D., ISIS Caliphate a threat to GCC. Pakistan Observer, 2014. Disponible en: http://pakobserver.net/detailnews.asp?id=246206

PRAHAN, Prasanta, The Kingdom and the Caliphate: Saudi Arabia's approach towards the Islamic State. Institute for Defence Studies and Analysis, ISDA Issue Brief, 2015.

RAMAZANI, R. KECHICHIAN, J., The Gulf Cooperation Council: Records and Analysis. University of Virginia, Virginia, 1988.

US assures GCC against external threat at summit. Middle East Monitor, 2015. Disponible en: https://www.middleeastmonitor.com/news/americas/18644-us-assures-gcc-againstexternal-threats-at-summit 
VELA, Justin, GCC to set up regional police force based in Abu Dhabi. The National, 2015. Disponible en: http://www.thenational.ae/world/gcc/gcc-to-set-up-regional-police-forcebased-in-abu-dhabi

WALTZ, Kenneth, Teoría de la política Internacional. Grupo Editor Latinoamericano (GEL), Buenos Aires, 1988.

YOUNG, Karen, The Politics of Small Slights in the GCC. The Washington Institute, 2014. 